\title{
Comparative Review on Computational Performance of Multistep Schemes in Solving One-Dimensional Linear Wave Equation
}

\author{
Serdar Hayytov ${ }^{1}$, Wah Yen Tey ${ }^{1,2,}{ }^{*}$, Hooi Siang Kang ${ }^{3}$, Mohammed W. Muhieldeen ${ }^{1}$, Omid Afshar ${ }^{4}$ \\ Department of Mechanical Engineering, Faculty of Engineering, UCSI University, Kuala Lumpur, Malaysia \\ Malaysia-Japan International Institute of Technology, Universiti Teknologi Malaysia, Kuala Lumpur, Malaysia \\ Marine Technology Center, School of Mechanical Engineering, Faculty of Engineering, Universiti Teknologi Malaysia, Malaysia \\ School of Engineering \& ICT, University of Tasmania, Australia
}

\section{ARTICLE INFO}

\section{Article history:}

Received 12 April 2021

Received in revised form 7 June 2021

Accepted 8 June 2021

Available online 11 June 2021
Keywords:

Wave equation; Multistep method; Computational performance

\section{ABSTRACT}

\section{Introduction}

There are many physical phenomena such as electromagnetics, structural vibration, acoustical propagation, ultrasonic cavitation, ocean wave propagation and fluid dynamics, which can be described via hyperbolic partial differential equations [1-7]. Formulating robust numerical schemes for solving hyperbolic equations are critical in simulating these complex phenomena, and it is still the interest of many researchers even today. Generally, there are two ways of solving hyperbolic models: one-step schemes and multistep schemes. One-step schemes are prevalent to be applied in solving hyperbolic models due to their simplicity. The examples of one-step scheme comprise Euler Explicit, Euler Implicit, and Generalised Trapezoidal Differencing (GTD) method. Euler explicit method is the most popular scheme, as it is very convenient to be applied.

\footnotetext{
* Corresponding author.

E-mail address: teywy@ucsiuniversity.edu.my (Wah Yen Tey)
}

https://doi.org/10.37934/cfdl.13.6.114 
Nonetheless, the associated problems of the Euler Explicit method are its limited CourantFriedrichs-Lewy (CFL) condition and computational burden in storing the history of field variables. El and GTD methods are dissipative and demanding high computational cost to solve the matrix system [8]. Moreover, these one-step schemes are of first-order accuracy, and the constraint can only be relieved by increasing the number of grids.

Several multistep schemes were proposed to enhance the numerical prediction. Most of the time, the order of accuracy is increased in multistep schemes. Thus, their computational efficiency is expected to be improved. The introduction of multistep schemes can be analogous to $p$-refinement in finite element method. Since the 1960s, multistep schemes for hyperbolic model have been introduced [9]. Typical examples of multistep schemes are two-step Lax-Wendroff (TSLW) method, MacCormack (MC) method, second-order Upwind (SOU) method, Rusanov-Burstein-Mirin (RBM) method, Warming-Kutler-Lomax (WKL) method, and fourth-order Runge-Kutta (RK4) method [1017]. TSLW, MC, and SOU method are two-step schemes, Rusanov and WKL method are three-step schemes, while RK4 contains four steps. Few early comparative works were reported by Anderson [18], Poloni et al., [19], and Liang et al., [20], but they are limited to the simulation of simple convective flow. General discussion on these methods can be found too in the work of Leer [9] and Pletcher et al., [21].

Some advanced multistep schemes are being developed recently by mathematicians to solve hyperbolic equations, particularly for complex structural dynamics accurately. These examples include Newmark method, Bathe method, implicit-explicit multistep method, differentialquadrature-based methods, Delfim-Soares method, Kim-Reddy method, and Kim's method [22-29]. Although they are robust in solving highly transient structural dynamics problems, increased computational cost will be incurred, especially when the problem domain has a higher dimension. Numerical accuracy shall not be overwhelmed by complexity in implementing an algorithm, depending on the necessity of the problem. Therefore, these advanced techniques are not our interest for the time being. In the current study, we consider a linear wave equation to assess the computational performance of multistep schemes.

Although multistep methods have been introduced since the 1960s, they are not as popular as the one-step Euler explicit method to be applied for engineering simulation [10-17]. Moreover, so far, there is no comprehensive comparative work reported on these multistep methods based on linear wave equation to the authors' knowledge. Therefore, the purpose of this paper is to systematically assess the computational performance of all multistep techniques, based on onedimensional second-order wave equation [10-17]. The assessment would provide general and practical information in selecting the most efficient possible scheme in simulating the second-order wave equation. The numerical accuracy and computational time of these methods will be compared in our study.

\section{Formulation of Numerical Solution}

The governing equation for one-dimensional wave propagation can be expressed as:

$$
\frac{\partial^{2} P}{\partial t^{2}}=c^{2} \frac{\partial^{2} P}{\partial x^{2}}
$$

where $P, t, c$, and $x$ represent scalar field variable, temporal domain, speed of wave and spatial domain, respectively. The equation is discretised via finite difference method according to different multistep methods. The initial and boundary condition of the problem can be expressed as: 


$$
\begin{aligned}
& P(x, 0)=0 \\
& P\left(x_{0}, t\right)=A \sin (2 \pi f t) \\
& \dot{P}\left(x_{N}, t\right)=0
\end{aligned}
$$

in which $A, f, N$ and $\dot{P}$ is scalar amplitude, frequency, total number of nodes, and time derivative of scalar, respectively. In the current simulation, $A, f$, and $N$ are set as $1,1 \mathrm{~Hz}$, and 71 , respectively. The length of the problem domain is $1 \mathrm{~m}$. The time step $(\Delta t)$ is obtained using Courant number $(\mathrm{Co})$, which can be defined as:

$$
\operatorname{Co}=\frac{c \Delta t}{\Delta x} \rightarrow \Delta t=\operatorname{Co}\left(\frac{\Delta x}{c}\right)
$$

where $\Delta x$ is the grid size. Perhaps, Courant number is an essential parameter to define the ratio between the time step and spatial resolution, administering the stability of numerical time marching. The details of the numerical formulations using are described in the following subsections [10-17]. The simulation is conducted using Intel ${ }^{\circledR}$ Core $^{\mathrm{TM}}$ i7-8700K CPU@3.70 GHz with RAM of 32 GB.

\subsection{Two-step Lax Wendroff Method}

Lax-Wendroff (LW) method was derived by Lax and Wendroff [10] in 1960 to solve hyperbolic equation for compressible flows, which involved discontinued time-dependent variables. The technique was then revised by Gadd [11] in 1978 to form a second-order accuracy two-step LW scheme, which was claimed to have higher performance without incurring cumbersome computation. The method was then improved by Carpenter [30] and Collins [31] to have third and fourth-order accuracy, respectively, with a cost of more complex numerical formulation. Jing et al., [32] optimised LW by introducing an optimised time-space-domain finite difference method for seismic imaging, which involves adaptive Courant number and higher-order finite differencing scheme. The applications of LW in solving fluid dynamics were reported in the work of Poloni et al., [19] and Serrano et al., [33]. Due to its efficiency, LW is integrated with other advanced numerical schemes such as discontinued Galerkin and discontinued Petrov-Galerkin method for complex finite element simulation [34-36]. The two-step LW method proposed by Gadd [11] will be investigated in the current work. The discretised wave equation formed via two-step LW method is:

Step 1: $P_{i+1 / 2}^{n+1 / 2}=\frac{1}{2}\left[\left(P_{i+1}^{n}+P_{i}^{n}\right)-\frac{c \Delta t}{\Delta x}\left(P_{i+1}^{n}-P_{i}^{n}\right)\right]$

Step 2: $P_{i}^{n+1}=P_{i}^{n}-\frac{c \Delta t}{\Delta x}\left(P_{i+1 / 2}^{n+1 / 2}-P_{i-1 / 2}^{n+1 / 2}\right)$

where $n, n+1 / 2$, and $n+1$ represent current time, intermediate time, and future time, respectively. Due to the existence of makeshift spatial and temporal domain, the actual grid number, and the total number of time stepping will be doubled. Note that for TSLW method, the predictor and corrector 
are using forward and backwards differencing, respectively, and the differencing could be used in a reversed way, depending on the boundary conditions of the problem [21].

\subsection{MacCormack Method}

MacCormack (MC) method was introduced by MacCormack [12,13], and it is popular among the scientific computing community, possibly due to its simplicity in dealing with highly convective flow without introducing additional terms as in LW method [21]. It is also named as predictor-corrector algorithm [37]. The applicability of MC method on highly advective flow has been discussed by Wendroff [38], Bernard [39], Jovanović and Djordjevic [40], and Hong [41]. The reported applications of $\mathrm{MC}$ method comprise solution on shallow water equation, advection-dispersion-reaction equations, heat equation, and Burgers' equation $[20,37,42,43]$. In general, $M C$ scheme can be formulated as:

Predictor: $\hat{P}_{i}^{n+1}=P_{i}^{n}-\frac{c \Delta t}{\Delta x}\left(P_{i+1}^{n}-P_{i}^{n}\right)$

Corrector: $P_{i}^{n+1}=\frac{1}{2}\left[P_{i}^{n}+\widehat{P}_{i}^{n+1}-\frac{c \Delta t}{\Delta x}\left(\widehat{P}_{i}^{n+1}-\widehat{P}_{i-1}^{n+1}\right)\right]$

in which $\hat{P}_{i}^{n+1}$ represent the predicted value for time $n+1$, and the value will be corrected in the subsequent step.

\subsection{Second-order Upwind Method}

Beam and Warming [14] tried to improve MC method by reversing the differencing of both predictor-corrector and increasing the order of accuracy of the corrector to form second-order upwind (SOU) method. Indeed, the SOU method is one of the most popular ways to solve complex fluid flow [44-46]. Many advanced techniques such as the flux corrector method, discontinuous Galerkin method, and weighted essentially non-oscillatory (WENO) method were further developed based on SOU Method [47-49]. Banks and Henshaw [50] extended the method to form a high-order upwind scheme, and this was later applied by Angel et al., [51] to simulate electromagnetic wave. In general, SOU method can be formed as in Eq. (6).

Predictor: $\widehat{P}_{i}^{n+1}=P_{i}^{n}-\frac{c \Delta t}{\Delta x}\left(P_{i}^{n}-P_{i-1}^{n}\right)$

Corrector: $P_{i}^{n+1}=\frac{1}{2}\left[P_{i}^{n}+\widehat{P}_{i}^{n+1}-\frac{c \Delta t}{\Delta x}\left(\hat{P}_{i}^{n+1}-\hat{P}_{i-1}^{n+1}\right)-\frac{c \Delta t}{\Delta x}\left(P_{i}^{n}-2 P_{i-1}^{n}+P_{i-2}^{n}\right)\right]$

\subsection{Rusanov-Burstein-Mirin Method}

Unlike LW, MC and SOU method, Rusanov-Burstein-Mirin (RBM) method involves three steps for computation of wave as illustrated as in Eq. (7) [15]: 
Step 1: $P_{i+1 / 2}^{(1)}=\frac{1}{2}\left(P_{i+1}^{n}+P_{i}^{n}\right)-\frac{1}{3} \frac{c \Delta t}{\Delta x}\left(P_{i+1}^{n}-P_{i}^{n}\right)$

Step 2: $P_{i}^{(2)}=P_{i}^{n}-\frac{2}{3} \frac{c \Delta t}{\Delta x}\left(P_{i+1 / 2}^{(1)}-P_{i-1 / 2}^{(1)}\right)$

Step 3: $P_{i}^{n+1}=P_{i}^{n}-\frac{c \Delta t}{\Delta x}\left(\frac{1}{24} F_{i}+\frac{3}{8} G_{i}+\frac{\omega}{24} H_{i}\right)$

where,

$F_{i}=-2 P_{i+2}^{n}+7 P_{i+1}^{n}-7 P_{i-1}^{n}+2 P_{i-2}^{n}$

$G_{i}=P_{i+1}^{(2)}-P_{i-1}^{(2)}$

$H_{i}=P_{i+2}^{n}-4 P_{i+1}^{n}+6 P_{i}^{n}-4 P_{i-1}^{n}+P_{i-2}^{n}$

$\omega$ is damping parameter in minimising the dissipative error, which can be defined based on the order of derivatives, in which its maximum value shall not exceed 3 [21]. The mathematical definition of $\omega$ for fourth and fifth derivatives can be optimised, as shown in Eq. (11) and Eq. (12), respectively.

$$
\begin{aligned}
& \omega=4\left(\frac{c \Delta t}{\Delta x}\right)^{2}-\left(\frac{c \Delta t}{\Delta x}\right)^{4} \\
& \omega=\frac{1}{5}\left[4\left(\frac{c \Delta t}{\Delta x}\right)^{2}+1\right]\left[4-\left(\frac{c \Delta t}{\Delta x}\right)^{2}\right]
\end{aligned}
$$

\subsection{Warming-Kutler-Lomax Method}

Warming et al., [17] tried to improve RBM method by replacing the first two steps of RBM scheme with MC scheme, and the derived formula can be presented as in Eq. (13):

Step 1: $P_{i}^{(1)}=P_{i}^{n}-\frac{2}{3} \frac{c \Delta t}{\Delta x}\left(P_{i+1}^{n}-P_{i}^{n}\right)$

Step 2: $P_{i}^{(2)}=\frac{1}{2}\left[P_{i}^{n}+P_{i}^{(1)}-\frac{2}{3} \frac{c \Delta t}{\Delta x}\left(P_{i}^{(1)}-P_{i-1}^{(1)}\right)\right]$

Step 3: $P_{i}^{n+1}=P_{i}^{n}-\frac{c \Delta t}{\Delta x}\left(\frac{1}{24} F_{i}+\frac{3}{8} G_{i}+\frac{\omega}{24} H_{i}\right)$

in which all the coefficients can be defined similarly with RBM method as in the previous subsection. 


\subsection{Fourth-order Runge-Kutta Method}

Fourth-order Runge-Kutta (RK4) method, as one of the most popular Runge-Kutta (RK) family solvers, is usually applied to solve ordinary differential equations (ODE). Besides hyperbolic equations, RK models are frequently used to solve parabolic models in fluid dynamics [52-54]. The details of the RK4 can be found in many textbooks [55]. However, partial differential equation (PDE) can be modified to resemble ODE, as demonstrated in Eq. (14a)-(14d). RK4 contains four steps in calculating the future variable.

Step 1: $P_{i}^{(1)}=P_{i}^{n}-\frac{c \Delta t}{2 \Delta x}\left(P_{i}^{n}-P_{i-1}^{n}\right)$

Step 2: $P_{i}^{(2)}=P_{i}^{n}-\frac{c \Delta t}{2 \Delta x}\left(P_{i}^{(1)}-P_{i-1}^{(1)}\right)$

Step 3: $P_{i}^{(3)}=P_{i}^{n}-\frac{c \Delta t}{\Delta x}\left(P_{i}^{(2)}-P_{i-1}^{(2)}\right)$

Step 4: $P_{i}^{n+1}=P_{i}^{n}-\frac{c \Delta t}{6 \Delta x}\left[\left(P_{i}^{n}-P_{i-1}^{n}\right)+2\left(P_{i}^{(1)}-P_{i-1}^{(1)}\right)+2\left(P_{i}^{(2)}-P_{i-1}^{(2)}\right)+\left(P_{i}^{(3)}-P_{i-1}^{(3)}\right)\right]$

\section{Results and Discussion}

The numerical solution for 1D wave equation using different multistep schemes can be illustrated as in Figure 1. Figure 1(a) and Figure 1(b) represent the scalar's distribution at Courant number 0.5 and 0.9, respectively, demonstrating the numerical accuracy and stability of investigated schemes. For comparison purpose, the exact solution for the boundary conditions as described as in the previous section is:

$P(x, t)=\sin \left(2 \pi t-\frac{2 \pi}{\lambda} x\right)$

where $\lambda$ is wavelength, which can be defined as the ratio between wave speed and wave frequency.

To further compare the features of multistep methods, Euler explicit solution is included too, which can be formed easily via Eq. (16).

$$
\frac{P_{i}^{n+1}-P_{i}^{n}+P_{i}^{n-1}}{\Delta t^{2}}=c^{2}\left(\frac{P_{i+1}^{n}-P_{i}^{n}+P_{i-1}^{n}}{\Delta x^{2}}\right)
$$

For low Courant number application, Euler explicit method performs as excellent as other multistep methods. However, Euler explicit method would have severe fluctuation when a high Courant number is applied, as shown in Figure 1(b). Moreover, despite its simplicity, Euler Explicit method calls for the longest computational time, as illustrated in Table 1. This shortcoming could be ascribable to the fact that the Euler explicit method has to store the previous and current time step's scalars before computing the future's one. Multistep methods do not need them. There is no 
significant difference in computational time between multistep schemes if the mesh number is small. In general, all multistep methods outperform the one-step Euler explicit method in terms of stability. From both Figure 1(a) and Figure 1(b), numerical dissipation and dispersion of scalar $P$ can be observed for all multistep methods, which is particularly critical for the RK4 method. Therefore, it is justifiable that RK4 is not widely applied to solve wave equation. Significant efforts are continuously made to improve RK4, which include the introduction of implicit RK methods, implicit-explicit RK method, and Runge-Kutta-Nyström methods [56-60].
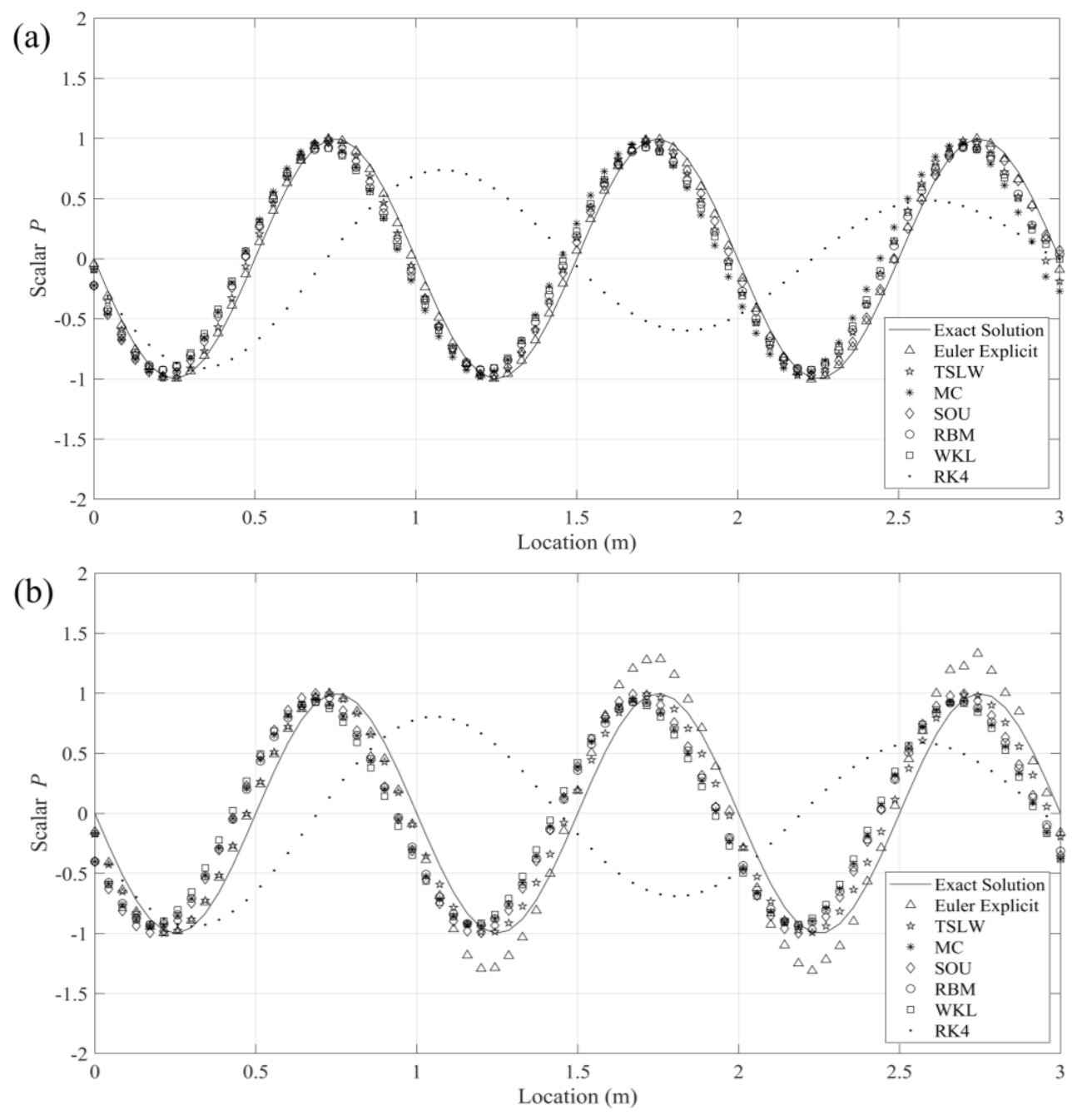

Fig. 1. Numerical solution for 1D wave equation using multistep schemes with grid number of 71 when Courant number is (a) 0.5 ; and (b) 0.9

Average numerical error is applied to examine the accuracy of different methods, in which the error can be calculated via Eq. (17). Euler explicit method can be regarded as having good accuracy despite its heavy computational time if the Courant number applied is small enough. The Courant number to be tested is 0.5 and 0.9 , which signifies the time marching speed which tends to be stable and unstable, respectively. At $\mathrm{Co}=0.5$ and 0.9 , SOU method and TSLW method has the best accuracy, respectively. Surprisingly, two-step methods (TSLW, MC and SOU) outperform three-step methods (RBM and WKL) and four-step method (RK4). To further examine these two-step methods, their numerical errors due to Courant number has been compared in Table 2. 
Average numerical error $=\frac{1}{N} \sum_{i=1}^{N}\left(\left|P_{i}^{\text {simulated }}-P_{i}^{\text {exact }}\right|\right)$

From Table 2, it has been proven that the one-step Euler explicit method has a minimal applicable Courant number, and it is unstable compared to other multistep processes. TSLW, MC, SOU, RBM and WKL method can be applied even at very high Courant number. Surprisingly, it is noteworthy that the SOU method does not diverge through the simulation until $\mathrm{Co}=1.9$. High-speed computing can be realised but with the loss of accuracy. Nonetheless, the drawback can be easily resolved by increasing the number of grids, as illustrated in Figure 2 and Table 3. From Table 3, SOU method performs excellently even at $\mathrm{Co}=1.5$ when the number of grids increases. For instance, with 12 seconds, SOU method can solve the problem of 201 grids with an average numerical error of 0.162633. Therefore, in general, two-step schemes, especially the SOU method, are excellent choices for fast and accurate hyperbolic problems.

\section{Table 1}

The computational time required for different methods for grid number of 71

\begin{tabular}{lll}
\hline Method & Courant Number & \\
& 0.5 & 0.9 \\
\hline Euler Explicit Method & $16.848 \pm 0.5 \mathrm{~s}$ & $8.540 \pm 0.5 \mathrm{~s}$ \\
TSLW Method & $11.745 \pm 0.5 \mathrm{~s}$ & $6.476 \pm 0.5 \mathrm{~s}$ \\
MC Method & $11.433 \pm 0.5 \mathrm{~s}$ & $6.637 \pm 0.5 \mathrm{~s}$ \\
SOU Method & $11.673 \pm 0.5 \mathrm{~s}$ & $6.293 \pm 0.5 \mathrm{~s}$ \\
RBM Method & $11.566 \pm 0.5 \mathrm{~s}$ & $6.288 \pm 0.5 \mathrm{~s}$ \\
WKL Method & $11.663 \pm 0.5 \mathrm{~s}$ & $6.259 \pm 0.5 \mathrm{~s}$ \\
RK4 Method & $11.572 \pm 0.5 \mathrm{~s}$ & $6.262 \pm 0.5 \mathrm{~s}$ \\
\hline
\end{tabular}

The results computed using RBM and WKL model in Figure 1 is based on the fifth derivatives of $\omega$, and the results could be worse if only the fourth derivative of $\omega$ is applied. For example, with the fourth derivatives of $\omega$, the RBM method's average error can be reduced to and for Co $=0.5$ and 0.9 , respectively. The existence of $\omega$, a free parameter, may lead to these numerical fluctuations [61]. Moreover, RBM scheme is still a centred scheme even though having a higher order of accuracy (as opposed to the non-centred scheme of MC and SOU method). Therefore, it inherits the limitations of the central finite difference model.

Burstein and Mirin [15] had suggested that RBM method is more appropriate for a smooth solution instead of discontinuous flow, and this remark was verified by Srinivas et al., [62] when they assessed the performance of RBM method for compressible flow. Gottlieb [63] proved that RBM is unconditionally unstable, especially in higher dimension problem. Simulation work by Darwis et al., [64] also illustrated that RBM method could not suppress shock instability. The complexity of RBM method is futile [9]. Due to these deficiencies, there is not much attention paid to RBM method, except for benchmarking and comparison purpose [65]. 


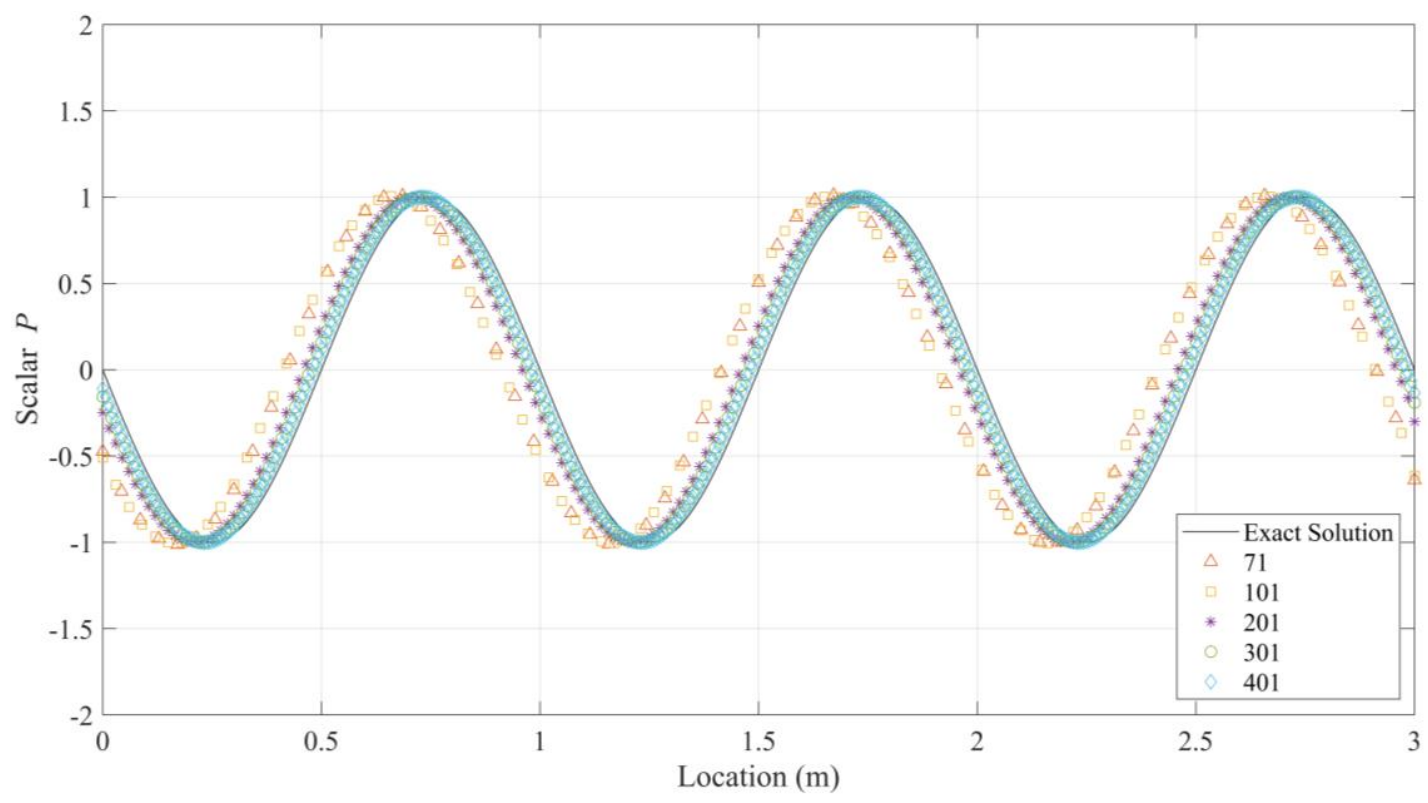

Fig. 2. Computation of linear wave equation using SOU method using different number of grids at $\mathrm{Co}=1.5$

\section{Table 2}

Average numerical errors of different methods compared with exact solution at different Courant number with grid number of 71

\begin{tabular}{llllllll}
\hline Co & EE & TSLW & MC & SOU & RBM & WKL & RK4 \\
\hline 0.5 & 0.04272 & 0.11308 & 0.19444 & 0.09301 & 0.1319 & 0.15378 & Dissipating \\
0.6 & 0.07627 & 0.10463 & 0.20159 & 0.11969 & 0.14715 & 0.17514 & Dissipating \\
0.7 & Diverged & 0.07759 & 0.190690 & 0.13149 & 0.147910 & 0.18101 & Dissipating \\
0.8 & Diverged & 0.11802 & 0.24400 & 0.21037 & 0.213060 & 0.25126 & Dissipating \\
0.9 & Diverged & 0.12281 & 0.26264 & 0.25414 & 0.24634 & 0.28918 & Dissipating \\
1.0 & Diverged & 0.05754 & 0.21504 & 0.22917 & 0.21504 & 0.26291 & Dissipating \\
1.1 & Diverged & Diverged & Diverged & 0.3542 & Diverged & Diverged & Dissipating \\
1.2 & Diverged & Diverged & Diverged & 0.37662 & Diverged & Diverged & Dissipating \\
1.3 & Diverged & Diverged & Diverged & 0.413740 & Diverged & Diverged & Dissipating \\
1.4 & Diverged & Diverged & Diverged & 0.41664 & Diverged & Diverged & Dissipating \\
1.5 & Diverged & Diverged & Diverged & 0.33526 & Diverged & Diverged & Dissipating \\
1.6 & Diverged & Diverged & Diverged & 0.38504 & Diverged & Diverged & Dissipating \\
1.7 & Diverged & Diverged & Diverged & 0.56024 & Diverged & Diverged & Dissipating \\
1.8 & Diverged & Diverged & Diverged & 0.24017 & Diverged & Diverged & Dissipating \\
1.9 & Diverged & Diverged & Diverged & 0.27335 & Diverged & Diverged & Dissipating \\
2.0 & Diverged & Diverged & Diverged & Diverged & Diverged & Diverged & Dissipating \\
\hline
\end{tabular}

Table 3

Average numerical errors and computational time for SOU method using different number of grids at $\mathrm{Co}=1.5$

\begin{tabular}{lll}
\hline Number of Grids & Average numerical error & Computational time $(\mathrm{s})$ \\
\hline 71 & 0.335258 & 3.970 \\
101 & 0.348415 & 5.387 \\
201 & 0.162633 & 11.502 \\
301 & 0.101223 & 17.780 \\
401 & 0.070699 & 23.110 \\
\hline
\end{tabular}

Although WKL method was modified based on the RBM method, WKL does not perform better than RBM all the time, depending on the flow types. WKL method is more suitable for inviscid 
Burger's equation but not discontinued flow $[18,66]$. Its complexity does not guarantee better performance, and hence the development of WKL is stagnated. If the advection term is not discretised reasonably, numerical accuracy is dubious even though higher-order differencing methods are applied.

Perhaps, all the analysis above (especially Table 1 ) is computed only for small mesh number ( $n=$ 71) while time for generating wave video is included in calculating the total computational. Big-O arithmetic analysis is further conducted to examine their algorithm efficiency, and the results can be shown as in Figure 3 . The investigation is conducted by setting $\mathrm{Co}=0.5$, while video recording for the wave is turned off. All the multistep schemes exhibit polynomial notation Nc, which means that the computational time increases in a polynomial manner with mesh resolution increment. $\mathrm{MC}$ records the fastest computational speed with the cost of high numerical error. TSLW will perform slightly slower than MC, yet with superior accuracy. The analysis further proved that time-consuming threestep algorithms could not reduce numerical errors compared with two-step methods.

In general, two-step algorithms are more robust compared with three-step or four step methods in computation of wave equation, and it is possible to be extended further for various applications related to wave fluctuations $[67,68]$.

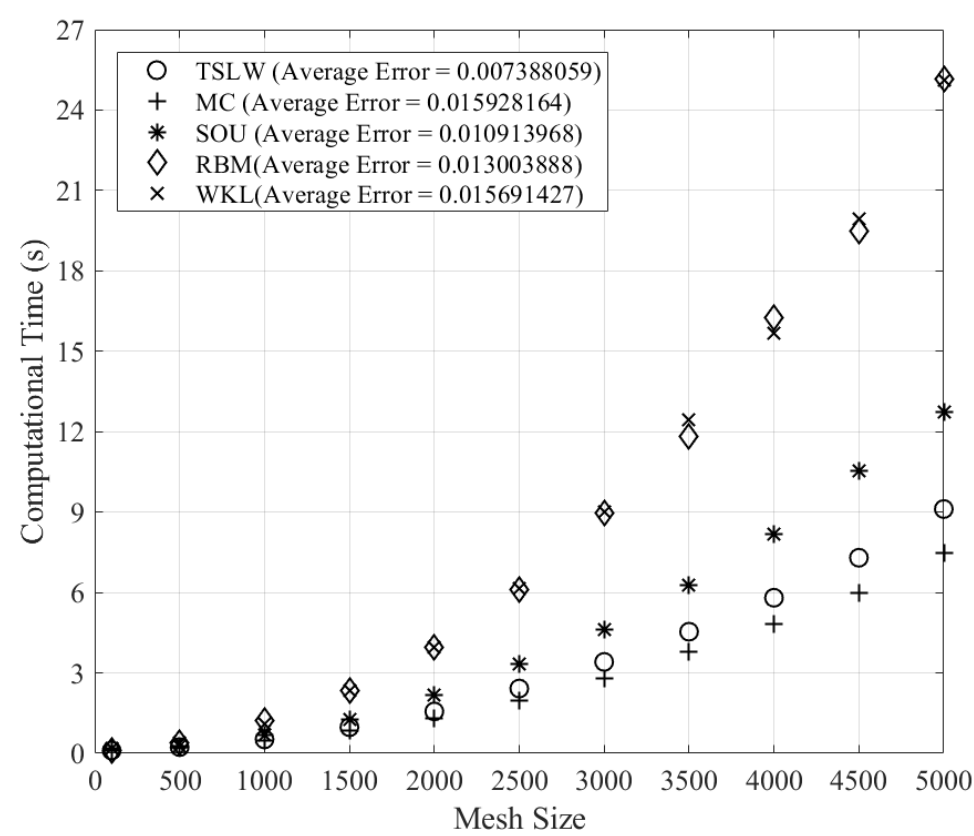

Fig. 3. The computational time for linear wave equation using different methods due to the varied number of grids at $\mathrm{Co}=$ 0.5

\section{Conclusion and Recommendations}

Multistep methods render broader Courant-Friedrichs-Lewy condition and faster computational speed than the single-step Euler explicit method, yet with the loose in avoiding numerical diffusion. Two-step methods (TSLW, MC and SOU) demonstrated excellent computational performance compared with three-step methods (RBM and WKL), while apparent numerical dissipation can be observed when RK4 method is used. Hence, among these multistep schemes, two-step schemes are recommended for the computation of hyperbolic wave equation. Among all two-step methods, TSLW slightly outperforms others. It is noteworthy that the SOU method can compute the solution excellently even at a high Courant number (not more than 1.9) if enough grids are assigned. The 
current investigation is somehow limited to one-dimensional study on wave equation. The performance and implementation of multistep schemes for the two-dimensional wave equation and fluid transport equations are interesting topics to be explored in the future, particularly with applications extended to engineering computational fluid dynamics.

\section{Acknowledgement}

The authors would like to thank the financial support from Centre of Excellence for Research, Value Innovation and Entrepreneurship (CERVIE), UCSI University for the research.

\section{References}

[1] Bathe, Klaus-Jürgen, Hou Zhang, and Yiguang Yan. "The solution of Maxwell's equations in multiphysics." Computers \& Structures 132 (2014): 99-112. https://doi.org/10.1016/j.compstruc.2013.09.006

[2] Takeshima, Masao, and Takumi Asakura. "Numerical simulation of vibration damping by granular materials." Applied Acoustics 162 (2020): 107189. https://doi.org/10.1016/j.apacoust.2019.107189

[3] Mattsson, Ken, Eric M. Dunham, and Jonatan Werpers. "Simulation of acoustic and flexural-gravity waves in icecovered oceans." Journal of Computational Physics 373 (2018): 230-252. https://doi.org/10.1016/i.jcp.2018.06.060

[4] Tey, Wah Yen, Habib Alehossein, Zonyi Qin, Kiat Moon Lee, Hooi Siang Kang, and Kee Quen Lee. "On stability of time marching in numerical solutions of rayleigh-plesset equation for ultrasonic cavitation." In IOP Conference Series: Earth and Environmental Science, vol. 463, no. 1, p. 012117. IOP Publishing, 2020. https://doi.org/10.1088/1755-1315/463/1/012117

[5] Lee, Joon Hin, Wah Yen Tey, Kiat Moon Lee, Hooi-Siang Kang, and Kee Quen Lee. "Numerical simulation on ultrasonic cavitation due to superposition of acoustic waves." Materials Science for Energy Technologies 3 (2020): 593-600. https://doi.org/10.1016/i.mset.2020.06.004

[6] Harun, Fatimah Noor. "Waves propagation in two-layer system over a non-rational submerged hump." In Journal of Physics: Conference Series, vol. 890, no. 1, p. 012032. IOP Publishing, 2017. https://doi.org/10.1088/17426596/890/1/012032

[7] Lerat, Alain. "A high-order time formulation of the RBC schemes for unsteady compressible Euler equations." Journal of Computational Physics 303 (2015): 251-268. https://doi.org/10.1016/i.jcp.2015.09.045

[8] Francesca, Priscilla, Wah Yen Tey, Lit Ken Tan, and M. W. Muhieldeen. "Investigation on Generalised Trapezoidal Differencing TimeMarching Scheme for Modelling of Acoustical Wave." CFD Letters 12, no. 2 (2020): 11-21.

[9] van Leer, Bram. "The development of numerical fluid mechanics and aerodynamics since the 1960s: US and Canada." In 100 Volumes of 'Notes on Numerical Fluid Mechanics', pp. 159-185. Springer, Berlin, Heidelberg, 2009. https://doi.org/10.1007/978-3-540-70805-6 14

[10] Lax, Peter, and Burton Wendroff. "Systems of Conservation Laws Comm." Pure and Applied Mathematics 13 (1960): 217-237. https://doi.org/10.1002/cpa.3160130205

[11] Gadd, A. J. "A numerical advection scheme with small phase speed errors." Quarterly Journal of the Royal Meteorological Society 104, no. 441 (1978): 583-594. https://doi.org/10.1002/qj.49710444104

[12] MacCormack, Robert William. "The effect of viscosity in hypervelocity impact cratering." In 4th Aerodynamic Testing Conference, p. 354. AIAA Paper, 1969. https://doi.org/10.2514/6.1969-354

[13] MacCormack, Robert William. "A numerical method for solving the equations of compressible viscous flow." AIAA Journal 20, no. 9 (1982): 1275-1281. https://doi.org/10.2514/3.51188

[14] Beam, R. M., and R. F. Warming. "Upwind second-order difference schemes and applications in aerodynamic flows." AlAA Journal 14, no. 9 (1976): 1241-1249. https://doi.org/10.2514/3.61457

[15] Burstein, Samuel Z., and Arthur A. Mirin. "Third order difference methods for hyperbolic equations." Journal of Computational Physics 5, no. 3 (1970): 547-571. https://doi.org/10.1016/0021-9991(70)90080-X

[16] Rusanov, V. V. "On difference schemes of third order accuracy for nonlinear hyperbolic systems." Journal of Computational Physics 5, no. 3 (1970): 507-516. https://doi.org/10.1016/0021-9991(70)90077-X

[17] Warming, R. F., P. Kutler, and H. Lomax. "Second-and third order noncentered difference schemes for nonlinear hyperbolic equations." AIAA Journal 11 (1973): 189-196. https://doi.org/10.2514/3.50449

[18] Anderson, Dale Arden. "A comparison of numerical solutions to the inviscid equations of fluid motion." Journal of Computational Physics 15, no. 1 (1974): 1-20. https://doi.org/10.1016/0021-9991(74)90065-5

[19] Poloni, M., D. E. Winterbone, and J. R. Nichols. "Comparison of unsteady flow calculations in a pipe by the method of characteristics and the two-step differential Lax-Wendroff method." International Journal of Mechanical Sciences 29, no. 5 (1987): 367-378. https://doi.org/10.1016/0020-7403(87)90118-4 
[20] Liang, Dongfang, Roger A. Falconer, and Binliang Lin. "Comparison between TVD-MacCormack and ADI-type solvers of the shallow water equations." Advances in Water Resources 29, no. 12 (2006): 1833-1845. https://doi.org/10.1016/i.advwatres.2006.01.005

[21] Pletcher, Richard H., John C. Tannehill, and Dale Anderson. Computational fluid mechanics and heat transfer. CRC press, 2012.

[22] Newmark, Nathan M. "A method of computation for structural dynamics." Journal of the Engineering Mechanics Division 85, no. 3 (1959): 67-94. https://doi.org/10.1061/JMCEA3.0000098

[23] Bathe, Klaus-Jürgen, and Mirza M. Irfan Baig. "On a composite implicit time integration procedure for nonlinear dynamics." Computers \& Structures 83, no. $31-32 \quad$ (2005): $2513-2524$. https://doi.org/10.1016/i.compstruc.2005.08.001

[24] Durran, Dale R., and Peter N. Blossey. "Implicit-explicit multistep methods for fast-wave-slow-wave problems." Monthly Weather Review 140, no. 4 (2012): 1307-1325. https://doi.org/10.1175/MWR-D-11-00088.1

[25] Bellman, Richard, and John Casti. "Differential quadrature and long-term integration." Journal of Mathematical Analysis and Applications 34, no. 2 (1971): 235-238. https://doi.org/10.1016/0022-247X(71)90110-7

[26] Soares Jr, Delfim. "A simple and effective new family of time marching procedures for dynamics." Computer Methods in Applied Mechanics and Engineering 283 (2015): 1138-1166. https://doi.org/10.1016/j.cma.2014.08.007

[27] Soares Jr, Delfim. "A novel family of explicit time marching techniques for structural dynamics and wave propagation models." Computer Methods in Applied Mechanics and Engineering 311 (2016): 838-855. https://doi.org/10.1016/i.cma.2016.09.021

[28] Kim, Wooram, and J. N. Reddy. "A New Family of Higher-Order Time Integration Algorithms for the Analysis of Structural Dynamics." Journal of Applied Mechanics 84, no. 7 (2017): 071008. https://doi.org/10.1115/1.4036821

[29] Kim, Wooram. "A new family of two-stage explicit time integration methods with dissipation control capability for $\begin{array}{llllll}\text { structural } \quad \text { dynamics." } & \text { Engineering } & \text { Structures } & 195 & \text { (2019): }\end{array}$ https://doi.org/10.1016/i.engstruct.2019.05.095

[30] Carpenter, K. M. "The accuracy of Gadd's modified Lax-Wendroff algorithm for advection." Quarterly Journal of the Royal Meteorological Society 107, no. 452 (1981): 467-470. https://doi.org/10.1256/smsqj.45214

[31] Collins, William G. "An accurate variation of the two-step lax-wendroff integration of horizontal advection." Quarterly Journal of the Royal Meteorological Society 109, no. 459 (1983): $255-261$. https://doi.org/10.1002/qj.49710945913

[32] Jing, Hao, Yushu Chen, Jian Wang, and Wei Xue. "A highly efficient time-space-domain optimized method with LaxWendroff type time discretization for the scalar wave equation." Journal of Computational Physics 393 (2019): 1 28. https://doi.org/10.1016/j.jcp.2019.04.066

[33] Serrano, J. R., F. J. Arnau, P. Piqueras, and O. García-Afonso. "Application of the two-step Lax and Wendroff FCT and the CE-SE method to flow transport in wall-flow monoliths." International Journal of Computer Mathematics 91, no. 1 (2014): 71-84. https://doi.org/10.1080/00207160.2013.783206

[34] Richter, Gerard R. "The discontinuous Galerkin method with diffusion." Mathematics of Computation 58, no. 198 (1992): 631-643. https://doi.org/10.1090/S0025-5718-1992-1122076-2

[35] Bürger, Raimund, Sudarshan Kumar Kenettinkara, and David Zorío. "Approximate Lax-Wendroff discontinuous Galerkin methods for hyperbolic conservation laws." Computers \& Mathematics with Applications 74, no. 6 (2017): 1288-1310. https://doi.org/10.1016/i.camwa.2017.06.019

[36] Zhao, Guozhong, and Xijun Yu. "The high order control volume discontinuous Petrov-Galerkin finite element method for the hyperbolic conservation laws based on Lax-Wendroff time discretization." Applied Mathematics and Computation 252 (2015): 175-188. https://doi.org/10.1016/i.amc.2014.12.024

[37] Zhang, Pei-Guang, and Jian-Ping Wang. "A predictor-corrector compact finite difference scheme for Burgers' equation." Applied Mathematics and Computation 219, no. $3 \quad$ (2012): $892-898$. https://doi.org/10.1016/j.amc.2012.06.064

[38] Wendroff, Burton. "The stability of MacCormack's method for the scalar advection equation." Applied Mathematics Letters 4, no. 2 (1991): 89-91. https://doi.org/10.1016/0893-9659(91)90176-V

[39] Bernard, Robert S. "A MacCormack scheme for incompressible flow." Computers \& Mathematics with Applications 24, no. 5-6 (1992): 151-168. https://doi.org/10.1016/0898-1221(92)90046-K

[40] Jovanović, Miodrag, and Dejana Djordjević. "Experimental verification of the MacCormack numerical scheme." Advances in Engineering Software 23, no. 1 (1995): 61-67. https://doi.org/10.1016/0965-9978(95)00053-T

[41] Hong, H. "The exact stability region of the MacCormack scheme for the scalar advection equation." Applied Mathematics Letters 9, no. 4 (1996): 99-101. https://doi.org/10.1016/0893-9659(96)00059-6

[42] Li, Guoyuan, and C. Rhett Jackson. "Simple, accurate, and efficient revisions to MacCormack and Saulyev schemes: high Peclet numbers." Applied Mathematics and Computation 186, no. 1 (2007): 610-622. https://doi.org/10.1016/i.amc.2006.08.008 
[43] Malinowski, L., and S. Bielski. "Transient temperature field in a parallel-flow three-fluid heat exchanger with the thermal capacitance of the walls and the longitudinal walls conduction." Applied Thermal Engineering 29, no. 5-6 (2009): 877-883. https://doi.org/10.1016/j.applthermaleng.2008.04.017

[44] Park, T. S., and Jang-Hyuk Kwon. "An improved multistage time stepping for second-order upwind TVD schemes." Computers \& Fluids 25, no. 7 (1996): 629-645. https://doi.org/10.1016/0045-7930(96)00014-X

[45] Xu, D., C. Shu, and B. C. Khoo. "Numerical simulation of flows in Czochralski crystal growth by second-order upwind QUICK scheme." Journal of Crystal Growth 173, no. 1-2 (1997): 123-131. https://doi.org/10.1016/S00220248(96)00791-9

[46] Tey, Wah Yen, Ryan Yuen Wye Hong, Yutaka Asako, Hooi Siang Kang, and Khai Ching Ng. "Analysis on computational efficiency of convection discretisation schemes in SIMPLE algorithm." Journal of Advanced Research in Fluid Mechanics and Thermal Sciences 58, no. 1 (2019): 100-117.

[47] Boris, Jay P., and David L. Book. "Flux-corrected transport. I. SHASTA, a fluid transport algorithm that works." Journal of Computational Physics 11, no. 1 (1973): 38-69. https://doi.org/10.1016/0021-9991(73)90147-2

[48] Cockburn, Bernardo, and Chi-Wang Shu. "The local discontinuous Galerkin method for time-dependent convectiondiffusion systems." SIAM Journal on Numerical Analysis 35, no. 6 (1998): $2440-2463$. https://doi.org/10.1137/S0036142997316712

[49] Jiang, Guang-Shan, and Chi-Wang Shu. "Efficient implementation of weighted ENO schemes." Journal of Computational Physics 126, no. 1 (1996): 202-228. https://doi.org/10.1006/icph.1996.0130

[50] Banks, Jeffrey W., and William D. Henshaw. "Upwind schemes for the wave equation in second-order form." Journal of Computational Physics 231, no. 17 (2012): 5854-5889. https://doi.org/10.1016/i.jcp.2012.05.012

[51] Angel, Jordan B., Jeffrey W. Banks, and William D. Henshaw. "High-order upwind schemes for the wave equation on overlapping grids: Maxwell's equations in second-order form." Journal of Computational Physics 352 (2018): 534-567. https://doi.org/10.1016/i.jcp.2017.09.037

[52] Zou, Shijun, Xijun Yu, and Zihuan Dai. "A Runge-Kutta discontinuous Galerkin method for Lagrangian ideal magnetohydrodynamics equations in two-dimensions." Journal of Computational Physics 386 (2019): $384-404$. https://doi.org/10.1016/i.jcp.2019.02.019

[53] Sanderse, Benjamin, and Arthur EP Veldman. "Constraint-consistent Runge-Kutta methods for one-dimensional incompressible multiphase flow." Journal of Computational Physics 384 (2019): 170-199. https://doi.org/10.1016/j.jcp.2019.02.001

[54] Komen, Ed MJ, Edo MA Frederix, T. H. J. Coppen, Valerio D'Alessandro, and J. G. M. Kuerten. "Analysis of the numerical dissipation rate of different Runge-Kutta and velocity interpolation methods in an unstructured collocated finite volume method in OpenFOAM ${ }^{\circledR} . "$ Computer Physics Communications 253 (2020): 107145. https://doi.org/10.1016/i.cpc.2020.107145

[55] Chapra, Steven C., and Raymond P. Canale. Numerical methods for engineers. Vol. 6. New York: Mcgraw-hill, 2010.

[56] Chen, Hao. "Kronecker product splitting preconditioners for implicit Runge-Kutta discretizations of viscous wave equations." Applied Mathematical Modelling 40, no. 7-8 (2016): $4429-4440$. https://doi.org/10.1016/j.apm.2015.11.037

[57] Liu, M. Y., L. Zhang, and C. F. Zhang. "Study on Banded Implicit Runge-Kutta Methods for Solving Stiff Differential Equations." Mathematical Problems in Engineering 2019 (2019). https://doi.org/10.1155/2019/4850872

[58] Weller, Hilary, Sarah-Jane Lock, and Nigel Wood. "Runge-Kutta IMEX schemes for the horizontally explicit/vertically implicit (HEVI) solution of wave equations." Journal of Computational Physics 252 (2013): 365-381. https://doi.org/10.1016/i.jcp.2013.06.025

[59] Zhao, Jingjun, Yu Li, and Yang Xu. "Multiderivative extended Runge-Kutta-Nyström methods for multi-frequency oscillatory systems." International Journal of Computer Mathematics 95, no. 1 (2018): $231-254$. https://doi.org/10.1080/00207160.2017.1366462

[60] Li, Jiyong, and Xinyuan Wu. "Energy-preserving continuous stage extended Runge-Kutta-Nyström methods for oscillatory Hamiltonian systems." Applied Numerical Mathematics $145 \quad$ (2019): $469-487$. https://doi.org/10.1016/j.apnum.2019.05.009

[61] Wesseling, P. "Accuracy of Third-Order Predictor-Corrector Difference Schemes for Hyperbolic Systems." AIAA Journal 10, no. 7 (1972): 948-949. https://doi.org/10.2514/3.50259

[62] Srinivas, K., J. Gururaja, and K. Krishna Prasad. "An assessment of the quality of selected finite difference schemes for time dependent compressible flows." Journal of Computational Physics 20, no. 2 (1976): 140-159. https://doi.org/10.1016/0021-9991(76)90060-7

[63] Gottlieb, David. "On the stability of Rusanov's third-order scheme." Journal of Computational Physics 15, no. 3 (1974): 421-426. https://doi.org/10.1016/0021-9991(74)90121-1 
[64] Darwis, Malina, Kamil Abdullah, and Akmal Nizam Mohammed. "Comparative study of Roe, RHLL and Rusanov fluxes for shock-capturing schemes." In IOP Conference Series: Materials Science and Engineering, vol. 243, no. 1, p. 012007. IOP Publishing, 2017. https://doi.org/10.1088/1757-899X/243/1/012007

[65] Roostaei, Morteza, Alireza Nouri, Vahidoddin Fattahpour, and Dave Chan. "Evaluation of numerical schemes for capturing shock waves in modeling proppant transport in fractures." Petroleum Science 14, no. 4 (2017): $731-745$. https://doi.org/10.1007/s12182-017-0194-x

[66] Islam, M. Rashidul, and M. Hanif Chaudhry. "Numerical solution of transport equation for applications in environmental hydraulics and hydrology." Journal of Hydrology 191, no. 1-4 (1997): $106-121$. https://doi.org/10.1016/S0022-1694(96)03077-6

[67] Ahmed, Mushtaq, Zafarullah Nizamani, Akihiko Nakayama, and Montasir Osman. "Some Recent Fluid-Structure Interaction Approaches for the Wave Current Behaviour With Offshore Structures." CFD Letters 12, no. 9 (2020): 15-26. https://doi.org/10.37934/cfdl.12.9.1526

[68] Tan, Wan Ching, Keng Wai Chan, Bee Mun Oon, How Tion Puay, and Yi Leang Lim. "Numerical Performance Study for the Buoy Shape of Point Absorber Wave Energy Converters." CFD Letters 12, no. 6 (2020): 118-133. https://doi.org/10.37934/cfdl.12.6.118133 